\title{
L’ancrage minéral du Vivant
}

\section{Mineral roots for the living world}

\author{
Thomas Heams ${ }^{1}$ \\ ${ }^{1}$ AgroparisTech - INRAE
}

RÉSUMÉ. Le paradigme dominant en biologie moléculaire assimile les êtres vivants à des machines de précision gouvernées par un programme génétique. Cette approche du vivant contribue à l'opposer radicalement au monde minéral, qui en semble l'antithèse. Pourtant, la recherche scientifique des origines de la vie vise à comprendre comment la vie est apparue dans un contexte entièrement minéral, et donc à tisser des liens plutôt qu'à scénariser des ruptures. Ce faisant, elle fait écho à plusieurs de nos mythes fondateurs, et en retour elle alimente la sciencefiction contemporaine, qui est peuplée d'étrange créatures hybrides entre la biologie et la minéralité. Or c'est aussi une voie que la science explore, où le vivant ne se serait pas arraché à la minéralité mais entretiendrait avec elle une relation réciproque, complexe et au long cours. Les conséquences épistémologiques de cette approche sont profondes, et contribuent à renouveler les définitions de la vie elle-même.

ABSTRACT. The dominant paradigm in molecular biology equates living things with precision machines ruled by a genetic program. This depiction of life contributes to radically oppose it to the mineral world, which seems to be its antithesis. However, Origins of Life studies aims to understand how life came to be in an entirely mineral context, and therefore to forge links rather than script ruptures. In so doing, it echoes many of our founding myths, and in turn it fuels contemporary science fiction, which is populated by strange hybrid creatures between biology and minerality. This is actually a path that science is also exploring, where the living would not be torn away from the minerality but would maintain a reciprocal, complex and long-term relationship with it. The epistemological consequences of this approach are profound, and contribute to renewing the definitions of life itself.

MOTS-CLÉS. Origines de la vie, minéral, vivant, science-fiction, définition de la vie, mythes, programme génétique, formes.

KEYWORDS. Origins of life, mineral, living, science fiction, definition of life, myths, genetic program, forms.

Qu'il soit permis à un biologiste de contribuer à un dialogue entre son objet d'étude, le vivant, et l'inerte, en l'occurence représenté par la minéralité de la céramique, est une précieuse opportunité. De prime abord, cela pourrait ressembler à un exercice de style, en particulier un jeu d'observation entre les contraires : mouvements, croissance, évolution du vivant semblant s'opposer en tout point au geste figé de l'autre, quand bien même il a la vitalité inouïe d'une céramique de Palissy. Le vivant, ses formes transitoires, ses individus changeants, traversés de flux génétiques, énergétiques, métaboliques, tout ceci serait une promesse d'avenir, là où le minéral serait advenu, en attente de son inéluctable dégradation. Oui, le vivant des biologistes d'aujourd'hui semble à bien des égards construit par opposition au monde minéral.

Pourtant ce rapport antithétique ne résiste pas à un examen rigoureux, et incite à se demander si cette construction est celle des faits, ou de notre regard sur eux. En premier lieu, parce que la question de la forme a longtemps été, dans la phase de modernisation de la biologie qui court tout au long du vingtième siècle, un impensé. Or, comme on va le voir en détail, c'est bien souvent en reconsidérant le rapport du vivant à la minéralité que cette question cruciale de la forme pourra se ressourcer. Mais avant d'y venir, d'où vient cet impensé, et que signifie-t-il vraiment ? Cette mise à l'écart de la question de la forme me semble principalement être un effet collatéral de la puissance heuristique de la génétique, à bien des égards le vaisseau amiral de la biologie du siècle passé. Sa capacité à expliquer la transmission et le maintien de structures adaptatives a en effet eu un prix, celui de reléguer au second plan le défi scientifique qu'est la compréhension du déploiement concret de ces formes dans chaque individu. À la suite des premiers généticiens du vingtième siècle naissant, les pionniers de la biologie moléculaire des années 1950-60 ont certes incroyablement bien compris le lien entre les gènes et les protéines, qui, par les interactions forme-dépendantes sont les 
briques des êtres vivants. Mais leur assemblage aux niveaux supérieurs et leur dynamique, l'édification progressive des organismes, les capacités de réparation comme de métamorphose de ceux-ci au cours de leur existence, restent encore bien souvent une boite noire qui témoigne qu'une des ambitions majeures de la biologie en tant que discipline, à savoir l'explication des formes vivantes, reste à l'état de promesse inachevée. Porté par la biologie moléculaire, le paradigme conquérant du "programme génétique » recouvre cette ambiguité d'un voile pudique et permet de faire d'une pierre deux coups : ce supposé programme sait décrire les mécanismes moléculaires élémentaires, mais on lui prête aussi, par anticipation, la capacité prochaine d'expliquer le développement, dans chaque individu, des formes macroscopiques attendues, à savoir son plan général d'organisation, ses tissus, ses organes. Au bilan, la grille de lecture génétique, donc informationelle, du vivant permettrait à la fois, de comprendre la transmission intergénérationnelles des caractères (la génétique elle-même), et l'apparentement des espèces les unes par rapport aux autres dans une perspective historique (la phylogénie), mais aussi la genèse dynamique des formes tridimensionnelles dans le vivant, de la molécule à l'individu. Lier les trois est crucial, car pour Charles Darwin, le fondateur de la théorie de l'évolution moderne, la biologie avait pour vocation impérieuse d'être une science des formes, et la théorie qui devait unifier les sciences du vivant devait prendre en charge cette mission à bras le corps. Malheureusement, force est de constater que jusqu'à présent, la relation, concrète et mécanistique, entre l'expression des gènes et la forme des vivants demeure extrêmement frustre, sans compter qu'elle s'adosse souvent au concept d'autoorganisation pour expliquer la genèse de ces formes, concept qui ne s'accorde que mal avec le primat du programme génétique.

On peut comprendre que, dans ce contexte d'une vie biologique principalement saisie comme un réseau complexe de flux d'informations, laissant la question de la forme de côté, son rapport à la minéralité soit perçu comme encore plus dérisoire, voire celui d'un anti-modèle. Comme me le résuma un jour un brillant biologiste, «comprendre la vie, c'est comprendre pourquoi il y a autre chose que des cailloux », résumant ainsi une position de principe finalement très consensuelle. Les minéraux ne respirent pas, ne mutent pas, ne se reproduisent pas, n'évoluent pas selon les dynamiques darwinienne (on verra plus loin que ces assertions méritent examen...) : quoi de plus éloigné a priori du monde vivant ? A y regarder de plus près, cette opposition pose en fait plus de problèmes qu'elle n'en résout. Le principal est que de dresser un trop grand fossé rend impossible une explication rationnelle et matérialiste des origines du vivant: si l'on veut produire un récit scientifique plausible de l'apparition de la vie sur Terre, il faut bien en passer par une explication qui tienne la route sur la transition qui s'est faite depuis la minéralité, et comment celle-ci a fourni la matière constitutive des premiers êtres vivants. Et moins cette transition est ponctuelle, c'est à dire plus la genèse du vivant se révèle un chemin partagé durable entre les vivants primordiaux et les minéraux, alors plus ce rapport au long cours doit s'expliquer en terme d'échanges dynamiques plutôt qu'en terme de séparation et d'autonomisation. Sans remonter aux temps primordiaux, il y a d'ailleurs une manière très simple de l'illustrer: puisque la vie est apparue de manière fugace et fragile, expliquer comment la biomasse totale sur Terre peut atteindre 500 milliards de tonnes équivalents carbone revient nécessairement à comprendre que le vivant s'ancre depuis son origine jusqu'à aujourd'hui dans la minéralité de notre planète, en en extrayant sa matière propre, au service de la production des myriades d'espèces biologiques qui constituent l'arbre du vivant. En outre, cette extraction n'est pas univoque, puisque les biomolécules se recylent certes au sein des vivants, mais aussi retournent à la terre, dans un processus réciproque de diversification : depuis que la vie est apparue sur Terre, on estime que 4300 espèces minérales nouvelles liées aux processus biologiques sont apparues, à mettre en regard des 500 à 1500 espèces qui préexistaient. Pour reprendre le terme du géologue Robert Hazen (2008), une évolution minérale existe donc bel et bien, et grâce à l'activité biologique, notre planète est, même sur le strict plan de sa géologie, la plus diversifiée que l'on connaisse. Enfin, ce rapport est aussi très intime, la minéralité ne se dissout pas en nous, mais y est présente, et fonctionnelle, à toutes les échelles : dans les os des vertébrés, dans les carapaces des arthropodes, et même au sein des molécules, puisqu'un tiers des protéines sont des 
métalloprotéines, c'est à dire qu'elles contiennent des atomes métalliques. La sélection naturelle sait retenir et exploiter les possibilités matérielles qu'offrent les minéraux, avec la même puissance qu'elle sait retenir des combinaisons de gènes adaptées à un environnement donné. Cette omniprésence de la minéralité chez les vivants doit nous conduire à un constat simple : le lien entre les minéraux et des vivants est un rapport de tension dynamique, et pas d'opposition.

Cette approche porte en elle la question de la limite entre le vivant et le non-vivant. Paradoxalement, c'est peut-être d'abord par les mythes et les fictions qu'il convient de chercher à la cerner. Les prendre au sérieux n'est pas une coquetterie de scientifique qui musarderait en dehors des chemins balisés de la rigueur expérimentale : mythes et fictions sont loin de se réduire à leur fonction de leur contribution à notre imaginaire, car ce sont aussi des indicateurs des intuitions et des cadres de pensées des époques qui les produisent. A cet égard, il est intéressant de voir que les origines minérales de l'homme sont pensées aussi bien dans la Genèse, où Adam est, étymologiquement, le glébeux (Chouraqui, 1987 ; Breton, 1995), c'est à dire le pétri fait d'argile, que dans la mythologie grecque où le titan Prométhée crée les hommes également d'eau et d'argile, au risque de la transgression divine. Plus près de nous, dans la tradition juive, le golem est une figure actualisée de cette même transgression, être d'argile porté à la vie par la ruse humaine. Cette référence convergente au même matériau malléable, minéral sans rigidité, il n'est pas interdit de la prendre comme une proposition somme toute pragmatique, non miraculeuse, de réponse à l'énigme de notre origine matérielle. Elle prend acte que les vivants proviennent de quelque chose, recyclent la matière du monde qui leur préexiste, et entérine donc une forme de continuité entre l'inorganique et l'organique. En outre, ici, l'argile est presque une passerelle, il nous préfigure par sa souplesse et ne s'oppose pas à nous à la manière d'un granite. Bien sûr, l'ingrédient crucial reste, dans ces récits mythiques, le souffle de la vie. Celui-là que précisément l'approche scientifique du vivant a délibérément mis de côté, et ce par quoi elle se différencie des mythes. Reste que la mise en scène de ce substrat matériel témoigne de la puissance de cette intuition dans notre ancrage au minéral. Et qu'il sera d'autant plus saisissant de constater que les feuillets d'argiles ont, comme nous le verrons plus bas, suscité toute l'attention des scientifiques modernes comme matrice possible des premières biomolécules.

Alors que le Frankenstein de Marie Shelley, sous-titré le Prométhée moderne, un des grands livres fondateurs de notre modernité, réactualisera au début du dix-neuvième siècle le mythe grec en délaissant l'argile pour la chair des cadavres, et en substituant l'étincelle électrique au feu sacré, une autre lignée d'auteurs va, entre le dix-neuvième et le vingtième siècle, reprendre le flambeau et creuser la veine fictionnelle de la vie minérale : les fondateurs européens de la science-fiction littéraire. Dans la littérature de langue française, c'est J.H. Rosny aîné, qui va a plusieurs reprises imaginer de telles formes de vies. Il y aura d'abord les Xipéhuz, en 1887, dans le roman court du même nom, qui sont des blocs rocheux mobiles qui menace des tribus humaines à l'aube de l'histoire. Les vies étranges nourriront d'autres de ses œuvres, comme dans Les Voyageurs de l'Infini, mais surtout en 1910 la Mort de la Terre, qui met en scène la survie des derniers humains, aux prises avec des Ferromagnétaux, êtres minéraux qui se repaissent des débris métalliques de la civilisation humaine, et vampirisent les humains eux-mêmes pour le fer de leur hémoglobine au point de provoquer leur extinction. L'idée, incroyablement romanesque, est aussi subtile d'un point de vue «scientifique». En effet la minéralité n'est pas ici construite comme une négation frontale de l'humanité, métonymie pour la vie au sens large : la relation métabolique proie-prédateur entre les deux espèces témoigne plus d'un rapport de co-dépendance, d'une compétition pour les ressources métalliques, et au final de la transformation de la matière vivante en une autre. Ce n'est donc ici pas tant la fin de la vie que la continuation de la vie sous d'autres possibilités matérielles. Ainsi ces Ferromagnétaux ne sont pas des entités venues de nulle part, ils entretiennent leur vitalité en s'appropriant une partie de ce qui nous constitue, matière et énergie. C'est d'ailleurs ce qui fait l'autre grande originalité de ce roman, relevée par Thomas Brandstetter (2012) dans un article auquel j'emprunte ici plusieurs références littéraires : proposer une hypothèse, se mettant en scène comme plausible, sur ce que sera notre finitude, la fin de notre espèce, et plus généralement la fin 
de la vie telle que nous la connaissons. Cette projection vers l'avenir tranche par son originalité avec les nombreux récits des origines, et enrichit d'une manière inédite l'imaginaire de la continuité entre le biologique et le minéral, continuité ici non plus seulement matérielle mais historique au sens le plus fort, car dans les deux directions temporelles. Ce qui incite implicitement à penser la vie biologique, telle que nous la connaissons, comme une parenthèse, une étrange et transitoire expérimentation dynamique du monde minéral. Ce faisant, Rosny aîné fait écho à un autre fondateur de la science-fiction moderne, le britannique H.G. Wells. Dans un article de 1894, Another basis for life (in Wells, 1975), l'auteur de la Guerre des Mondes qui était aussi un essayiste scientifique des mieux informés de son temps, avait spéculé sur la possibilité d'une vie reposant non pas sur le carbone mais sur le silicium (un des atomes les plus abondants sur Terre, plus lourd que le carbone mais possédant avec lui plusieurs propriétés physico-chimiques), une hypothèse qui, régulièrement jusqu'à nos jours, a fait passionnément débat au sein de la communauté scientifique qui cherche des formes de vie ailleurs : les exobiologistes (pour ce débat, voir Bains, 2004). Ceci témoigne de la fécondité de la science-fiction, non pas tant comme divertissement (ce qu'elle est aussi, et heureusement), mais comme laboratoire d'hybridation où l'imaginaire fournit des pistes au réel. La thématique des minéraux vivants a fourni des œuvres variées, comme la Révolte des Pierres de Léon Groc en France (1930), ou Der gläserne Regen d'Annie Francé-Harrar en Allemagne (1948), qui chacun décrivent des minéraux vivants d'origine lunaire, ou A Martian Odyssey (1934) et The Red Peri (1935) de l'américain Steven Weinbaum, dont les protagonistes sont des entités minérales et vivantes respectivement martienne et plutonienne. Bien que très disparates, ces œuvres ont toutes en commun de questionner, de manière centrale ou non mais toujours présente, la distinction claire entre le minéral et le vivant, et à envisager des formes intermédiaires. Cette hypothèse, on le verra est plus qu'une curiosité, mais bien plus un défi épistémologique de première importance.

À ces mondes fictionnels fait écho, bien sûr, une approche philosophique, naturaliste, scientifique, qui s'est construite depuis les Lumières, puisque c'est au moins dès les écrits du philosophe Maupertuis que celle-ci commence à se mettre en place. Dans la Vénus Physique (1745) il cherche à tisser des liens entre les formes des concrétions minérales nommées « Arbre de Diane », qu'on peut produire au laboratoire par des réactions chimiques d'oxydoréduction, et celle de structures vivantes comme un fotus. Les biologistes parmi les plus influents du siècle suivant, comme les fondateurs de la théorie cellulaire, Theodor Schwann et Matthias Jakob Schleiden, et plus tard August Weismann, le fondateur du néodarwinisme vont travailler la question du rapport entre le vivant et le minéral. Certains vont même s'y perdre, comme Ernst Haeckel qui ira jusqu'à parler de l'Âme des cristaux, titre d'un de ses ouvrages tardifs (1917). William Preyer, enfin, dans les années 1880 , proposa un des premiers cadres théoriques d'explications des origines du vivant, envisageant une Terre primordiale initialement brûlante qui, en se refroidissant, aurait cristallisé et provoqué la sortie du protoplasme à l'origine des êtres vivants (analysé in Oparine, 1924). Même si cette thèse est désuète et repose sur un vitalisme problématique, elle illustre que le lien au minéral s'imaginait alors à tous les niveaux d'échelle. Loin d'une opposition frontale, l'historien de la biologie J. Lorch (1974) souligna au contraire le « charisme des cristaux ", selon le titre d'un de ses articles, auprès des biologistes du dix-neuvième siècle.

Et contrairement aux apparences ce charisme fut durable, même si le vingtième siècle, on l'a vu plus haut, est, pour le meilleur et le pire, le siècle du gène selon l'expression de l'historienne Evelyn Fox Keller (2003), de la redécouverte des lois de Mendel en 1900 au séquençage du génome humain en 2001, en passant par la synthèse évolutive moderne dans les années 1930-1940 (c'est-à-dire la convergence entre la théorie darwinienne de l'évolution et la génétique), la découverte de la structure de l'ADN en 1953, et les succès retentissants de la biologie moléculaire. Quelle place, dans cet univers informationnel, quasi dématérialisé, pour le dialogue avec le minéral ? De manière contre-intuitive, il s'est bel et bien poursuivi, quoique plus discrètement. Les biologistes de synthèse du vingt-et-unième siècle, ceux qui cherchent à transformer la vie radicalement, voire à la fabriquer en laboratoire, et qui sont souvent considérés à tort ou à raison comme l'avant-garde de la bio-ingénierie, citent paradoxalement, comme l'un de leur prédécesseurs, un médecin nantais qui serait probablement retourné à l'anonymat s'il n'avait pas eu la bonne fortune d'écrire, en 1912, un ouvrage au titre préfigurateur : la Biologie Synthétique. Dans cet ouvrage, Stéphane Leduc questionne la frontière entre le vivant et l'inanimé, et aborde la question sous l'angle de la forme, avec une approche 
originale. En résonance avec Maupertuis, il va notamment demeurer célèbre par ses «jardins (ou paysages ) chimiques », des concrétions minérales produites en laboratoire de manière inorganique mais dont l'apparence, la forme, peut tromper et faire penser à du vivant. Leduc n'était pas dupe et ne prétendait surtout pas que ces productions étaient vivantes, mais elles étaient son point d'appui pour justement se questionner sur la bonne manière de distinguer le vivant du non vivant. Il fut un des premiers à envisager formellement des formes intermédiaires possibles, et ses travaux, bien que largement obsolètes sur le plan expérimental, continuent d'alimenter intellectuellement les questionnements contemporains sur le vivant. Un exemple peut l'illustrer: à l'été 1996, une poussée de fièvre se produisit dans le monde scientificomédiatique. La NASA soutint l'hypothèse qu'une météorite d'origine martienne, ALH84001, avait révélé au microscope des structures ressemblant à des bactéries, bien que de tailles très inférieures à celles connues sur Terre. Cette annonce fracassante faisait suite à des décennies de débats sur les modalités de recherche de vie ailleurs que sur Terre, notamment sur Mars. A défaut de petits hommes verts, que devait-on chercher : de l'eau, du mouvement, des cellules, des polymères ? Chacune de ces réponses est d'ailleurs possible, avec ses avantages et ses inconvénients, même si toutes partent d'un principe paradoxal : chercher de l'inconnu en espérant qu'il ressemble au connu. L'épisode de 1996 tranchait à sa manière le débat : c'est bien la forme, l'aspect de ces microstructures, qui, ici, se trouvait l'argument majeur de nature à appuyer l'hypothèse de l'existence d'une vie (même fossile) ailleurs que sur Terre. Ce qui n'était, en somme, pas grand-chose de plus que du Leduc revisité. Ce le fut jusqu'au bout d'ailleurs, puisque Leduc utilisait ses jardins chimiques non pas comme une preuve de vie, mais à l'inverse comme une incitation à la prudence face aux apparences: l'origine biogénique des nanostructures nichées dans ALH84001 n'ayant jamais pu être démontrée autrement que par cette apparence, et la possibilité de produire ces formes de manière abiotique ayant aussi, à l'inverse, été rappelée, alors on finit progressivement par admettre que ce n'était encore pas cette fois qu'on prouverait l'existence de la vie sur Mars.

Ainsi, le legs de Leduc enjambe largement son siècle. Mais l'arrimage du vivant au monde minéral ne s'est pas arrêté dans ses jardins chimiques. Sans aucunement prétendre à l'exhaustivité, quelques exemples cardinaux me semblent l'illustrer. Les deux premiers concernent les Origines du Vivant. Cette question est devenue une discipline scientifique en soi, même si elle est caractérisée par des modalités de véridiction tout à fait spécifique : face à l'impossibilité de remonter effectivement dans le temps au(x) moment(s) de ces origines, les spécialistes des origines du vivant combinent approches expérimentales et observations naturalistes pour proposer des scénarios plausibles et, idéalement, le moyen de les comparer entre eux. L'un de ces scénarios, dit de la relève génétique, et porté par le chimiste Alexander Graham Cairns-Smith depuis les années 1960 envisage que les biomolécules comme l'ADN ou l'ARN aient pu prendre la place de molécules précédentes de nature géochimique, notamment au sein des feuillets d'argile, qui créent des espaces en (quasi) deux dimensions favorisant les rencontres moléculaires et les catalyses (Cairns-Smith, 1982). Un autre scénario plus récent, défendu par Eugene Koonin et William Martin depuis une quinzaine d'année, explore la possibilité que les premières «cellules» soient apparues au sein de petites bulles minérales, en fait des anfractuosités dans un minéral comme la serpentinite. Nourris par les flux de matière et d'énergie en provenance des sources hydrothermales, des bouillons de cultures retenus dans ces bulles, plus ou moins communicantes entre elles, auraient pu donner naissance aux premières réactions métaboliques, tout comme premiers systèmes génétiques qui, chacun, auraient pu s'y développer, se sophistiquer (Koonin \& Martin, 2005). Le contenu de ces bulles aurait pu ne s'autonomiser que dans un second temps, dans des vésicules autonomes et mobiles, parentes de nos cellules modernes : la première phase de ce scénario illustre qu'une vie génétique, métabolique, et pour autant minérale est pensable. Chacun de ces scénarios a ses ardents défenseurs, et des critiques nombreux, mais ils soulignent à leur manière la plausible ancestralité de notre rapport intime avec les minéraux.

Par ailleurs, plus on connaît les cristaux, plus il est difficile de les penser comme inertes. L'épistémologue Mark Bedau (1991), souligne que des cristaux d'argile ont des propriétés de reproduction, de variations, d'hérédité, et même d'adaptativité. Or prises toutes ensembles, ces propriétés sont très souvent considérée comme le paradigme du vivant ! Enfin, impossible de ne pas souligner que la molécule la plus emblématique de la vie terrestre, l'ADN, est elle même une structure cristalline. La compréhension de sa structure, découverte en 1953 par Francis Crick, Rosalind Franklin et James Watson, confirma les prédictions d'Ernst Schrödinger avait parlé, dans son célèbre livre What is life? (1944) d'un cristal apériodique qui, selon lui, devait inéluctablement être découvert pour expliquer le stockage et l'exploitation de l'information génétique. Beaucoup a été écrit, à très juste titre, sur la place démesurée qu'a pris l'ADN dans nos représentations du vivant. On en fait à tort le centre de la vie, de son origine même, on résume trop souvent la vie à la 
génétique, que ce soit dans les débats sociétaux sur l'aide à la procréation, ou dans les peurs du clonage ou de l'eugénisme. Reste que l'ADN est une molécule cruciale, qui d'ailleurs est à l'interface entre la génétique d'une part, mais aussi le métabolisme (une des quatre bases de l'ADN, L'ATP, est aussi une molécule clé des échanges énergétiques dans la cellule). Les acides nucléiques, que sont l'ADN et l'ARN, sont des marqueurs de toute la vie connue sur Terre. N'y a-t-il pas là un savoureux clin d'oeil que cette molécule censée incarner la vitalité qui nous extirperait de notre cocon minéral, soit elle-même un cristal ?

Au terme de ces évocations, dont je mesure l'aspect fragmentaire, que nous dit ce compagnonnage entre le vivant et le minéral ? On l'a esquissé à plusieurs reprises, il peut donc être le temps de l'aborder de front : c'est principalement la question de la limite entre le vivant et le non-vivant qui est ici posée. Même si c'est une des questions les plus anciennes, ce n'est pas qu'une question théorique, elle a des répercussions concrètes. Protéger la vie, protéger la biodiversité par exemple, présuppose que l'on sache ce qu'est la vie biologique, ce qui en fait l'originalité. Or, la porosité entre le vivant et le minéral met cette distinction au défi. La vie ressortirait-elle de cet apeîron, (souvent traduit par indéfinissable) ce principe premier si cher à Anaximandre, l'un des plus anciens promoteur d'une observation rationnelle de la nature (Rovelli, 1995)? Une manière de sortir de cette ornière est d'accepter que la définition du vivant ne soit pas une délimitation. A la suite de philosophes, de biologistes expérimentaux et d'historiens de la biologie, j'ai proposé que l'on fasse le deuil d'une catégorie d'objets de l'univers qui seraient «vivants » par opposition à tous les autres. Mais pour autant, je n'ai pas voulu renoncer à la nécessité de définir la vie biologique, et à ces fins je me suis référé notamment à des formalisations théoriques du philosophe Mark Bedau (2012) déjà croisé plus haut, qui montre que même avec quelques caractéristiques simples, comme le métabolisme, la génétique et la compartimentation cellulaire, on peut imaginer des centaines de manières intermédiaires d'être vivant, entre le pur inerte et la vie «pleine», telle que nous la connaissons. Les interactions que ces trois caractéristiques peuvent avoir entre elles, par les rapports d'interdépendance qu'elles peuvent nouer de manière pérenne, forment une combinatoire très étoffée de possibles. Si l'on accepte cette prémisse, on peut alors envisager qu'il existe non pas une mais des centaines de manières possibles d'être vivant, à toutes les échelles. Ironiquement, cela permet de retrouver l'autre traduction possible de l'apeîron d'Anaximandre, l'illimité, et de l'assumer résolument: l'absence de délimitation entres ces vies étranges bouscule notre vision de la biosphère et de l'originalité intrinsèque du vivant, mais non pas comme un problème. C'est au contraire, une invitation, par le biais de cette épistémologie expérimentale qui part des faits biologiques pour questionner nos cadres d'interprétation, à reconsidérer plus largement notre manière de définir les objets du monde, avec d'autres outils que les catégories strictes. J'ai proposé de nommer infravies ces vies étranges, qu'elles soient théoriques, observées dans la nature, fabriquées en laboratoire (Heams, 2019). Toutes, néanmoins, sont caractérisées par un principe organisateur, la mise en mouvement adaptative de la matière. Et toutes, sans exceptions, ne trouvent leur pérennité que dans un rapport de tension fécond avec le monde minéral.

\section{Références}

Bains W. (2004), « Many chemistries could be used to build living systems », Astrobiology.

Bedau M.A. (1991), « Can biological teleology be naturalized ? », The Journal of Philosophy.

Bedau MA (2012), «A functional account of degrees of minimal chemical life », Synthese.

Brandstetter T (2012), «Imagining inorganic life : Crystalline aliens in science and fiction », in Geppert A. (eds), Imagining Outer Space, Palgrave Macmillan.

Breton P (1995), À l'image de l'Homme, Seuil.

Cairns-Smith AG (1982), Genetic Takeover and the Mineral Origins of Life, Cambridge University Press.

Chouraqui E. (trad.) (1987) La Bible, Desclée de Brouwer.

Fox Keller E (2003) Le Siècle du gène, Gallimard

Hazen RM, Papineau D, Bleeker W, Downs RT, Ferry F, McCoy T, Sverjensky D, Yang H. (2008), « Mineral evolution », American Mineralogist.

Heams T (2019) Infravies, le vivant sans frontières, Seuil

Koonin EV, Martin W (2005), « On the origin of genomes and cells within inorganic compartments », Trends in Genetics. 
Lorch J (1974), «Charisma of crystals in biology», in Elkana Y. (éd.), The Interaction Between Science and Philosophy, Humanities Press.

Oparine A (1924), Proiskhozhdenie zhizni, Izd. Moskovskii Rabochii ; trad. fr. (1965), L'Origine de la Vie, Masson. Rovelli C (2015) Anaximandre de Milet ou la naissance de la pensée scientifique, Dunod.

Schrödinger E. (1944), What is life ?, Cambridge University Press ; trad. fr. (1986), Qu'est-ce que la vie ?, Seuil.

Wells HG (1975) Early writings in Science and Fiction, University of California Press 